\title{
Computational Investigation of the Effect \\ of Air Injection into the Fluid Flow \\ with Precessing Vortex Core
}

\author{
Artem A. Dekterev and Dmitriy A. Dekterev* \\ Institute of Thermophysics SB RAS \\ 1 Lavrentev, Novosibirsk, 630090, Russia
}

Received 00.06.2016, received in revised form 00.08.2016, accepted 00.11.2016

The paper is devoted to the numerical study of the effect of air injection into the hydrodynamic vortex chamber at the flow characteristics with precessing vortex core. The calculation results are in good agreement with the experimental data obtained at the Institute of Thermophysics SB RAS. Obtained numerical data confirm the effect of the decrease of the vortex precession frequency at low amount of air and further smooth growth of the precession frequency with increasing gas flow rate in the stream.

Keywords: computational fluid dynamics, precessing vortex core, two-phase flows.

\section{Численное исследование влияния ввода воздуха}

\section{В жидкостный поток}

с прецессирующим вихревым ядром

\author{
А.А. Дектерев, Д.А. Дектерев \\ Институт теплофизики \\ им. С.С. Кутателадзе СО РАН
}

Россия, 630090, Новосибирск, пр. Академика Лаврентьева, 1

Статья посвящена численному исследованию влияния впрыска воздуха в гидродинамическую вихревую камеру на характеристики потока с прецессирующим вихревым ядром. Результать расчетов хорошо согласуются с экспериментальными данными, полученными в Институте теплофизики СО РАН, и подтверждают эффект резкого уменьшения частоты прецессии вихря при вводе малого количества воздуха и дальнейшего плавного роста частоты прецессии при увеличении газосодержания в потоке.

(c) Siberian Federal University. All rights reserved

* Corresponding author E-mail address: dek art@mail.ru 
Ключевые слова: вычислительная гидрогазодинамика, прецессирующее вихревое ядро, двухфазные потоки.

\section{Введение}

Закрученные потоки нередко встречаются в природе и технике. Так, например, формирование закрученных течений происходит в следе самолетных и гребных винтов, ветрогенераторов, рабочих колес ГЭС и пр. $[1,2]$. На основе закрутки рабочей среды работают такие устройства, как циклоны, сепараторы, вихревые расходомеры и т.д. Полезные свойства закрученных течений широко применяются в теплоэнергетике, например, с ее помощью добиваются стабилизации пламён в горелочных устройствах. Однако использование закрученных потоков с параметром крутки выше некоторого критического осложняется возникновением в них сложных нестационарных вихревых структур, таких как, например, прецессирующее вихревое ядро (ПВЯ).

Прецессирующее вихревое ядро служит одним из механизмов возникновения пульсаций в гидротурбинах. Это явление возникает за рабочим колесом, когда поток жидкости после прохождения через турбину имеет достаточно большую остаточную закрутку. Возникающие при этом пульсации давления представляют серьезную опасность: они вызывают сильные вибрации конструкции гидротурбины, которые в случае резонанса могут привести к разрушению оборудования. Пульсации давления могут также воздействовать на процессы кавитации, усиливая кавитационную эрозию.

Исследованию явления ПВЯ посвящено множество расчетных и экспериментальных работ. В работах [1-9] исследуются условия формирования, частотные характеристики, параметры зон рециркуляции, полей и профилей скорости и прочие характеристики течения с формированием ПВЯ применительно к различным техническим приложениям. В ряде работ [1-5] отмечается линейная зависимость частоты прецессии вихря от расхода среды в широком диапазоне чисел Рейнольдса и при различных методах организации закрутки потока. Отмечается, что один из наиболее актуальных вопросов - возможность управления параметрами ПВЯ, в частности изменения частоты прецессии с целью ухода от нежелательных режимов работы агрегатов.

В статье [7] уделяется внимание конструктивным способам подавления низкочастотных пульсаций давления в отсасывающей трубе гидротурбины. Отмечается, что для снижения динамических нагрузок и расширения зоны нормальной работы гидротурбины в мировой практике используются различные конструктивные решения, позволяющие повлиять на уровень динамических явлений в проточной части: установка крестовины в конус отсасывающей трубы, удлинение конуса обтекателя рабочего колеса (РК), установка рёбер на стенки конуса отсасывающей трубы, дозированный впуск воздуха под РК, установка вертушки на конус обтекателя рабочего колеса и др. Одним из наиболее эффективных методов подавления пульсаций давления при работе гидротурбины в режиме частичной нагрузки служит установка рёбер на стенки конуса отсасывающей трубы. Использование рёбер способствует диссипации энергии закрутки потока при неоптимальных режимах работы турбины и таким образом позволяет уменьшить пульсации давления, вызванные вихревым жгутом. При режимах, близких к максимальной мощности, применение рёбер малоэффективно, так как в таком случае вихревой 
жгут располагается близко к оси диффузора и рёбра уже не способствуют разрушению структуры вихревого жгута и диссипации энергии закрутки потока в конусе отсасывающей трубы. Здесь для стабилизации потока предпочтительнее использовать впуск воздуха под рабочим колесом.

\section{Постановка задачи}

В работах [3, 8], проведенных в Институте теплофизики СО РАН, исследуется влияние впуска воздуха в гидродинамическую систему на характеристики прецессирующего вихревого ядра. Отмечается, что увеличение процента впускаемого воздуха $Q_{g}$ при различных расходах воды в целом приводит к росту частоты прецессии $f$, однако при достаточно малом содержании второй фазы (на примере расходов воды $Q_{w}=1.9$ и $Q_{w}=3.4$ ) наблюдается обратный эффект уменьшения частоты, как это видно на рис. 1.

Основываясь на экспериментальных данных Института теплофизики СО РАН, было проведено численное моделирование задачи. Главными целями стало тестирование современных математических моделей применительно к подобным задачам, а также численное подтверждение особенностей влияния второй фазы на частотные характеристики прецессирующего вихря, включая случай с малым содержанием второй фазы.

За основу для проведения численных расчетов была взята конструкция экспериментального стенда Института теплофизики СО РАН $[3,8]$. Установка представляет собой цилиндрическую вихревую камеру с лопаточным завихрителем на входе и крестовиной, разбивающей закрученный поток, в конусной области на выходе.

Форма завихрителя и геометрия расчетной области представлены на рис. 2. Для вычислений брали неструктурированную расчетную сетку с многогранными ячейками. Параметр крутки, определенный по геометрическим параметрам завихрителя [1], равнялся $S=1.1$. В качестве несущей фазы использовалась вода, дисперсная газовая фаза (воздух) задавалась равномерно распределенной по входу в виде пузырьков диаметром 100 мкм.

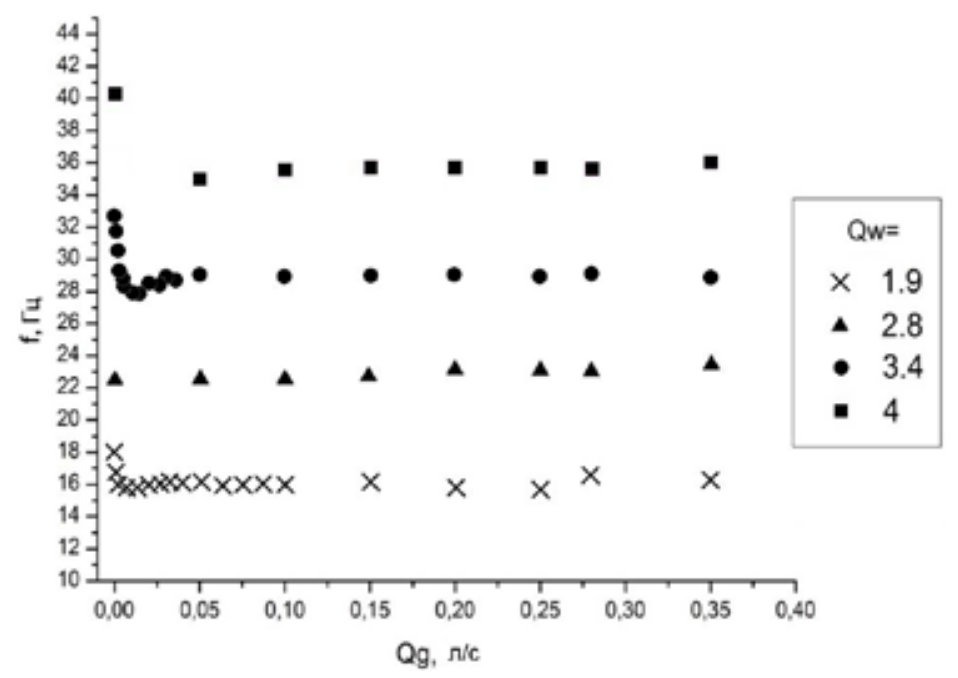

Рис. 1. Зависимость частоты ПВЯ от объема инжектируемого воздуха [8] 


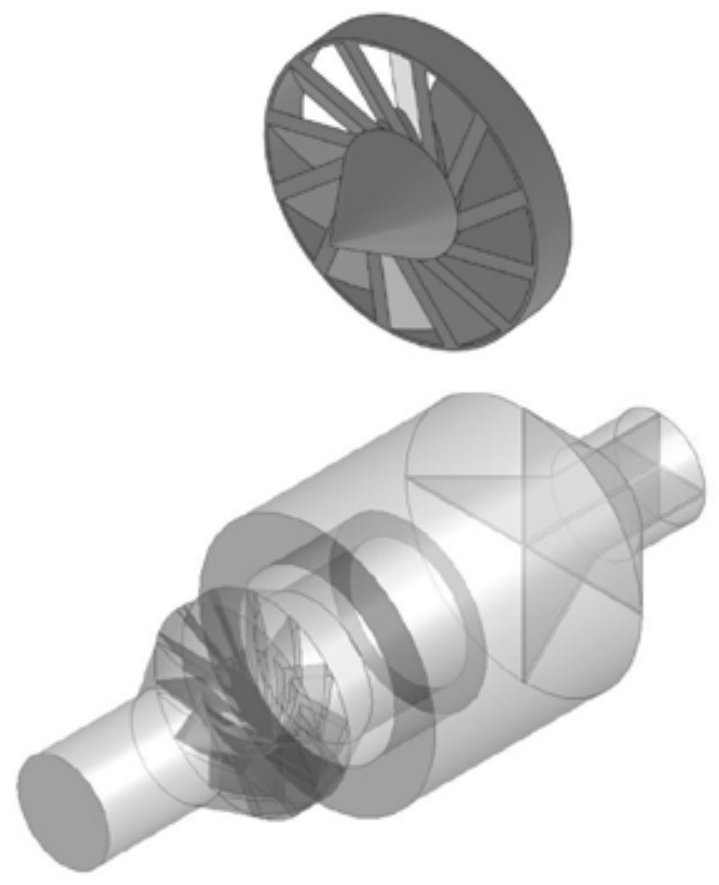

Рис. 2. Расчетная геометрия

В связи с тем что турбулентные закрученные вихревые течения - одни из наиболее сложных видов течений, остро встает вопрос об их корректном математическом моделировании. Существует множество методов численного моделирования сложных гидро- и газодинамических задач на основе различных моделей турбулентности. Так, в работе [9] проводится тестирование разных методов и моделей для описания закрученного течения с формированием ПВЯ. В частности, в качестве тестовой задачи рассматривается течение закрученного потока в круглом канале с внезапным расширением. Тестовые расчеты показывают значительное преимущество метода отсоединенных вихрей (DES) над RANS (уравнения Навье-Стокса, осредненные по Рейнольдсу) по качеству описания течения. В то же время DES не требует больших вычислительных мощностей, как метод моделирования крупных вихрей (LES). Этим и обосновывается дальнейшее использование DES для моделирования течения с прецессирующим вихревым ядром.

\section{Математическая модель}

Для численного моделирования нестационарного турбулентного течения несжимаемой жидкости использовали уравнения Рейнольдса:

$$
\begin{aligned}
& \nabla \cdot v=0, \\
& \frac{\partial \rho v}{\partial t}+\nabla(\rho v v)=-\nabla p+\nabla \cdot\left(\tau^{m}+\tau^{t}\right),
\end{aligned}
$$

где тензор вязких напряжений 


$$
\tau_{i j}^{m}=\mu\left(\frac{\partial v_{i}}{\partial x_{j}}+\frac{\partial v_{j}}{d x_{i}}\right),
$$

где $\tau^{t}$ - тензор рейнольдсовых напряжений.

Для замыкания уравнений применяли метод отсоединенных вихрей DES (detached eddy simulation) на основе модели турбулентности Спаларта-Аллмараса. Этот подход хорошо себя зарекомендовал для расчетов нестационарных закрученных течений [2]. В модели Спаларта-Аллмараса решается уравнение на модифицированную вязкость, которое имеет вид

$$
\begin{aligned}
& \frac{\partial(\rho \widetilde{v})}{\partial t}+\nabla \cdot(\rho V \widetilde{v})=\frac{1}{\sigma} \nabla[(\mu+\rho \widetilde{v}) \nabla \widetilde{v}]+ \\
& +\frac{C_{b 2} \rho}{\sigma}(\nabla \widetilde{v})^{2}+C_{b 1} f_{r 1} \rho \widetilde{S} \widetilde{v}-C_{w 1} \rho f_{w}\left(\frac{\widetilde{v}}{d}\right)^{2}
\end{aligned}
$$

B DES - версии масштаб $d$ заменяется на

$$
\widetilde{d}=\min \left(d, C_{d e s} \Delta\right),
$$

где $C_{d e s}$ - эмпирическая константа, а $\Delta$ определяется как максимальный из трех размеров контрольного объема $\Delta_{x}, \Delta_{y}, \Delta_{z}$. Таким образом, в пограничных слоях работает RANS-модель, а в остальной области - LES.

Для моделирования газовой фазы использовали два метода, один из них - это диффузионноинерционная модель (ДИМ), реализованная в следующем виде:

уравнение переноса массовой доли дисперсной фазы

$$
\frac{\partial \rho r_{\beta}}{\partial t}+\nabla\left(\rho v_{\alpha} \cdot r_{\beta}\right)=\nabla\left(\Gamma_{\beta} \cdot \nabla r_{\beta}\right)-\nabla\left(\rho r_{\beta} u^{r}\right)
$$

коэффициент турбулентной диффузии второй фазы

$$
\Gamma_{\beta}=\frac{\mu_{t}}{S c_{\beta}} \text { или } \Gamma_{\beta}=\left(1-r_{\beta}\right) \frac{\mu_{t}}{S c_{\beta}} .
$$

Относительная скорость второй фазы связана с дрейфовой скоростью соотношением (в случае одной дисперсной фазы)

$$
u^{r}=\left(1-r_{\beta}\right) u_{\beta \alpha} .
$$

Относительная скорость второй фазы

$$
u_{\beta \alpha}=\tau_{p}\left(g-\frac{\partial v}{\partial t}-\left(v_{\alpha} \cdot \nabla\right) v_{\alpha}\right)
$$

где время релаксации и функция трения

$$
\tau_{p}=\frac{d_{\beta}{ }^{2}}{18 \mu_{\alpha} f_{\text {drag }}}\left(\rho_{\beta}-\rho_{\alpha}\right),
$$




$$
f_{\text {drag }}=\left\{\begin{array}{cc}
1+0.15 \mathrm{Re}^{0.687}, \mathrm{Re} \leq 1000 \\
0.0183, & \operatorname{Re}>1000
\end{array} .\right.
$$

Относительное число Рейнольдса:

$$
\operatorname{Re}=\frac{\rho_{\alpha} d_{\beta}\left|u_{\beta \alpha}\right|}{\mu_{\alpha}} .
$$

Второй подход моделирования дисперсной фазы - это полная двухжидкостная модель, основанная на подходе Эйлера. Для каждой из фаз решается система уравнений. Уравнение сохранения импульса:

$$
\frac{\partial}{\partial t}\left(r_{\alpha} \rho_{\alpha} v_{\alpha}\right)+\nabla \cdot\left(r_{\alpha}\left(\rho_{\alpha} v_{\alpha} v_{\alpha}\right)\right)=-r_{\alpha} \nabla p_{\alpha}+\nabla \cdot\left(r_{\alpha} \mu_{\alpha}\left(\nabla v_{\alpha}+\left(\nabla v_{\alpha}\right)^{T}\right)+M_{\alpha}\right.
$$

$M_{\alpha}$ описывает межфазные силы, действующие на фазу $\alpha$ со стороны других фаз. Уравнение неразрывности

$$
\frac{\partial}{\partial t}\left(r_{\alpha} \rho_{\alpha}\right)+\nabla \cdot\left(r_{\alpha} \rho_{\alpha} v_{\alpha}\right)=0
$$

Уравнение сохранения объемной доли

$$
\sum_{\alpha=1}^{N_{p}} r_{\alpha}=1
$$

Фазы имеют одно общее поле давления

$$
p_{\alpha}=p \text { для всех } \alpha=1, \ldots \ldots, N_{p} .
$$

Сила межфазного сопротивления, действующая на фазу $\alpha$ со стороны фазы $\beta$,

$$
\begin{aligned}
& M_{\alpha}=c_{\alpha \beta}^{d}\left(v_{\beta}-v_{\alpha}\right), \\
& c_{\alpha \beta}^{d}=\frac{C_{D}}{8} A_{\alpha \beta} \rho_{\alpha}\left|v_{\beta}-v_{\alpha}\right|,
\end{aligned}
$$

где $\rho$ - плотность; $\left(U_{\alpha}-U_{\beta}\right)$ - относительная скорость; $\alpha$ - сплошная фаза; $\beta$ - дисперсная фаза. Плотность межфазной поверхности записывается как

$$
A_{\alpha, \beta}=\frac{6 \widetilde{r}_{\beta}}{d_{\beta}},
$$

где

$$
\widetilde{r}_{\beta}=\left\{\begin{array}{c}
\max \left(r_{\beta}, r_{\min }\right) \quad \text { if }\left(r_{\beta}<r_{\max }\right) \\
\max \left(\frac{1-r_{\beta}}{1-r_{\max }} r_{\max }, r_{\min }\right) \quad \text { if }\left(r_{\beta}>r_{\max }\right)
\end{array}\right.
$$

Для коэффициента сопротивления использовалась корреляция Шиллера-Неймана: 


$$
C_{D}=\max \left(\frac{24}{\operatorname{Re}}\left(1+0.15 \mathrm{Re}^{0.687}\right), 0.44\right) .
$$

Для моделирования турбулентности для двухфазной задачи решаются те же самые уравнения, что и для одной фазы, но плотность и вязкость берутся для смеси. Для решения задачи использовались собственный пакет программ SigmaFlow и коммерческий пакет Fluent. Для аппроксимации конвективных членов уравнений применялась схема QUICK. Связь поля скорости и давления осуществлялась посредством алгоритма SIMPLEC. Система линейных алгебраических уравнений решалась при помощи многосеточного решателя. Временной шаг составлял $0.001 \mathrm{c}$, применялась неявная схема второго порядка точности.

\section{Результаты}

На первом этапе исследований для тестирования моделей течения несжимаемой жидкости проводились нестационарные расчеты однофазного течения. Расход воды варьировался в пределах от 1 до 3 л/с. На рис. 3 представлен график зависимости частоты прецессии вихревого ядра от расхода воды. Видно, что зависимость носит линейный характер. Результаты расчета очень хорошо коррелируют с экспериментальными данными.

Для сравнения двух подходов моделирования дисперсной фазы проводились серии нестационарных расчетов двухфазного течения. Расход воды составлял 1.17 л/с, расход воздуха на входе варьировался в пределах от 0 до 1 л/с.

Анализ рассчитанных полей концентраций показал, что, как и наблюдалось в эксперименте, газовая фаза собирается в центре вихревого жгута, образуя воздушную полость (рис. 4).

Сравнение данных расчетов двухфазных течений на основе разных моделей показало, что полная двухжидкостная модель (модель Эйлера) и дифузионно-инерционная модель (ДИМ) дают очень близкие результаты зависимости частоты прецессии от расхода воздуха (рис. 5).

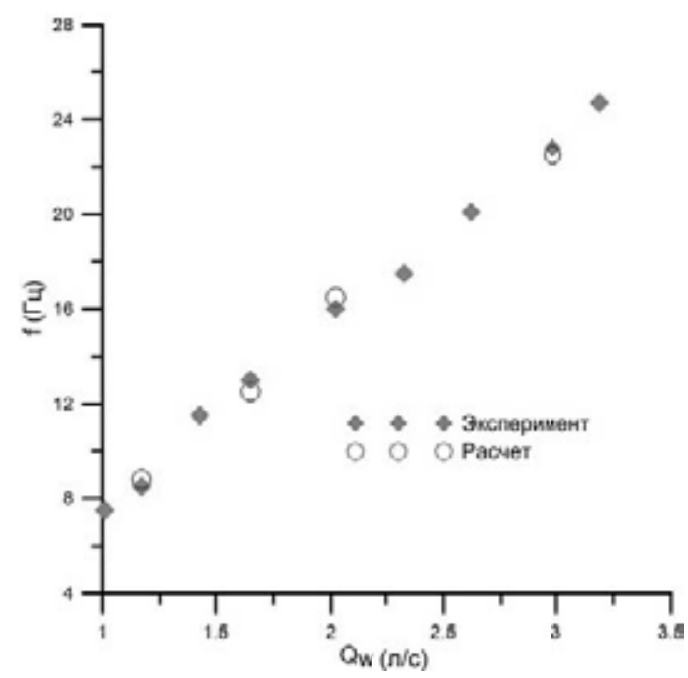

Рис. 3. График зависимости частоты прецессии от расхода воды 


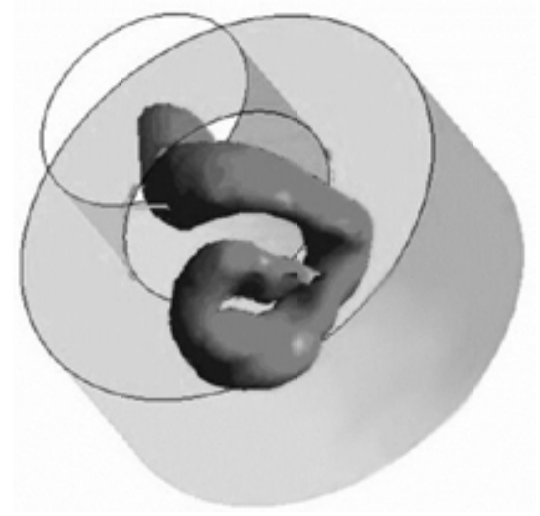

Рис. 4. Визуализация вихревого ядра изоповерхностью плотности

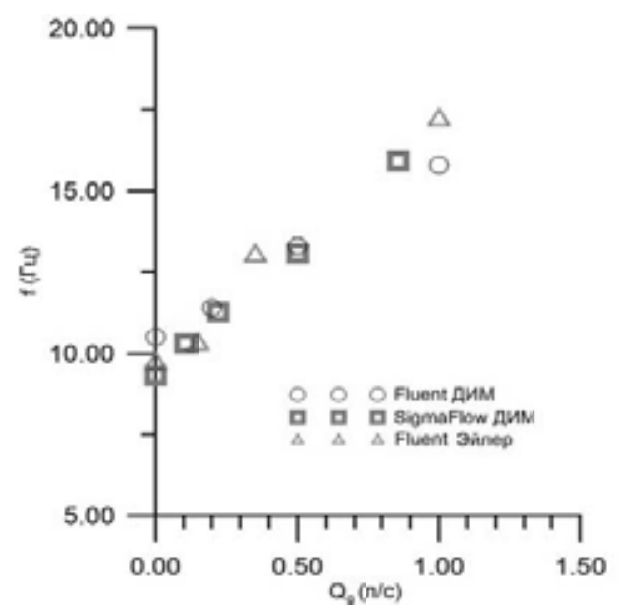

Рис. 5. Зависимость частоты прецессии от расхода газа

Из представленного графика видно, что по мере увеличения газосодержания наблюдается равномерное увеличение частоты прецессии. Результаты, полученные с использованием программ SigmaFlow и Fluent, хорошо согласуются между собой.

Более детальное исследование области малых газосодержаний также дало результат, близкий к экспериментальному. В данном случае расход воды составлял 2.02 л/с, расход воздуха на входе варьировался в пределах от 0 до 0.5 л/с. Действительно, как видно на рис. 6 , частота прецессии вихря при однофазном течении несколько выше частоты прецессии с малыми содержаниями второй фазы.

\section{Заключение}

По результатам данной работы можно сделать вывод, что метод отсоединенных вихрей DES хорошо подходит для расчета сложных турбулентных закрученных течений с образованием прецессирующего вихревого ядра. Также показана адекватность применения диффузионноинерционной модели и полной модели двухжидкостной модели Эйлера для описания двухфаз- 


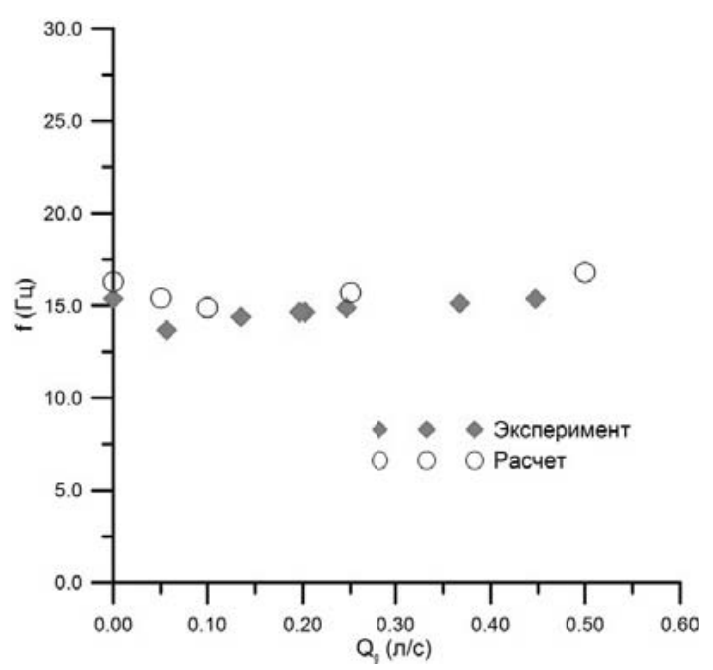

Рис. 6. Зависимость частоты прецессии от расхода газа при малых содержаниях второй фазы

ных явлений в таких течениях. Результаты численного моделирования подтвердили линейную зависимость частоты ПВЯ от расхода, полученную в экспериментах. Кроме того, подтвержден эффект влияния газовой фазы на частотные характеристики ПВЯ, включая случай с малыми газосодержаниями.

«Расчетные исследования выполнены при частичной поддержке гранта РФФИ 16-48240707 p_a."

\section{Список литературы}

[1] Гупта А., Лилли Д., Сайред Н. Закрученные потоки. М.: Мир, 1987. 590 с. [Gupta A., Lilli D., Saired N. Swirling flows. Moscow, Mir, 1987. 590 p. (in Russian)]

[2] Алексеенко С.В., Куйбин П.А., Окулов В.Л. Введение в теорию концентрированных вихрей. Новосибирск: Изд-во Ин-та теплофизики СО РАН, 2003. 504 с. [Alekseenko S.V., Kuibin P.A., Okulov V.L. Introduction to the theory of concentrated vortices. Novosibirsk, In-t tepolofiziki SO $R A S, 2003.504$ p.]

[3] Алексеенко С.В., Куйбин П.А., Окулов В.Л., Шторк С.И. Прецессия вихря в газожидкостном потоке. Тепломассообмен и гидродинамика в закрученных потоках [The precession of the vortex gas-liquid flow. Heat and mass transfer and hydrodynamics in swirling flows (in Russian)]

[4] Litvinov I.V., Shtork S.I., Kuibin P.A., Alekseenko S.V., Hanjalic K. Experimental study and analytical reconstruction of precessing vortex in a tangential swirler. International Journal of Heat and Fluid Flow, 2013, 42, 251-264.

[5] Дектерев, Д.А.; Дектерев, А.А.; Шторк, С.И. Экспериментальное и численное исследование прецессирующего вихревого ядра в условиях сильной закрутки потока. Журнал Сибирского федерального университета. Техника и технологии, 2012, 5(5), 487-494 [An Experimental and a Numerical Study of Precessing Vortex Core in the High Swirl Number Flow Conditions. J. Sib. Fed. Univ. Eng. technol., 2012, 5(5), 487-494 (in Russian)] 
[6] Шторк С.И., Комас О., Фернандес Э.К., Хейтор М.В. Аэродинамическая структура нестационарного закрученного потока позади внезапного расширения. Теплофизика и аэромеханика, 2005, 12(2) [Shtork S.I., Komas O., Fernandes E.K., Kheitor M.V. The aerodynamic structure of unsteady swirling flow behind the sudden expansion. Thermophysics and Aeromechanics, 2005, 12(2) (in Russian)]

[7] Семёнов Г.А., Смирнова А.Ю., Дектерёв А.А., Минаков А.В., Сентябов А.В. Численное исследование конструктивных способов подавления низкочастотных пульсаций давления в отсасывающей трубе гидротурбины. Труды Академэнерго, 2013, 3, 26-40 [Semenov G.A., Snirnova A.Iu., Dekterev A.A., Minakov A.V., Sentiabov A.V. Numerical study of constructive methods for the suppression of low-frequency pressure pulsations in the draft tube of hydraulic turbine. Trudy Akademenergo, 2013, 3, 26-40 (in Russian)]

[8] Литвинов И.В. Влияние газовой фазы на частоту прецессии вихревого ядра. Устойчивость и турбулентность течений гомогенных и гетерогенных жидкостей, 2010 [Litvinov I.V. The effect of the gas phase at the frequency of precession vortex core. Stability and turbulence of flows of homogeneous and heterogeneous liquids, 2010 (in Russian)]

[9] Сентябов А.В., Гаврилов А.А., Дектерев А.А. Исследование моделей турбулентности для расчета закрученных течений. Теплофизика и аэромеханика, 2011, 1, 81-93 [Sentiabov A.V., Gavrilov A.A., Dekterev A.A. Investigation of turbulence models for calculation of swirling flows. Thermophysics and Aeromechanics, 2011, 1, 81-93 (in Russian)]

[10] Минаков А.В., Гаврилов А.А., Дектерев А.А. Моделирование закрученного турбулентного течения в отсасывающей трубе гидротурбины Усть-Илимской ГЭС. Сборник тезисов VI Всероссийского семинара по теплофизике и теплоэнергетике. Красноярск, 2009, с. 71 [Minakov A.V., Gavrilov A.A., Dekterev A.A. The simulation of swirling turbulent flow in the draft tube of hydraulic turbine Ust-Ilimskiy HPP. Abstracts of VI all-Russia seminar on Thermophysics and heatpower engineering. Krasnoyarsk, 2009, p. 71 (in Russian)] 